\title{
APPLICATION OF LASER SCANNING SURVEYING TO ROCK SLOPES RISK ASSESSMENT ANALYSIS
}

\author{
M. Corsetti ${ }^{\text {a }}$, P.J.V. D'Aranno ${ }^{\text {a }}$, M. Marsella ${ }^{\text {a }}$, S. Scifoni ${ }^{\text {a }}$, A. Sonnessa ${ }^{\text {a }}$ \\ ${ }^{a}$ DICEA, Dept. of Civil, Construction and Environmental Engineering, University of Roma La Sapienza, Italy
}

\begin{abstract}
:
The methods for understanding rock instability mechanisms and for evaluating potential destructive scenarios are of great importance in risk assessment analysis dedicated to the establishment of appropriate prevention and mitigation actions. When the portion of the unstable rock mass is very large, effective actions to counteract the risks are complex and expensive. In these conditions, an optimal risk management cannot ignore procedures able to faster and accurately acquire i) geometrical data for modeling the geometry of the rock walls and implementing reliable forecasting models and ii) monitoring data able to describe the magnitude and the direction of deformation processes. These data contributes to the prediction of the behavior of a landslide if the measurements are acquired frequently and reliable numerical models can be implemented. Innovative geomatic techniques, based on GPS, Terrestrial Laser Scanning Surveying (TLS), automated total station and satellite and ground SAR Interferometry, have been recently applied to define the geometry and monitoring the displacements of unstable slopes. Among these, TLS is mainly adopted to generate detailed 3D models useful to reconstruct rock wall geometry by contributing to the estimation of geo-mechanical parameters, that is orientation, persistence and apparent spacing of rock discontinuities. Two examples of applications of TLS technique to the analysis of a large front in a quarry and of a rock shoulder of a dam are presented.
\end{abstract}

KEY WORDS: Terrestrial Laser Scanning, rock slope surveying, monitoring

\section{INTRODUCTION}

Rock instabilities represent a significant issue for hydrogeological risk assessment which require demanding scientific and technical capabilities both for the monitoring and for the modeling aspects. The methods for controlling rock landslides able to support mitigation actions should be based on integrated system including in-situ geotechnical and topographical measurements and non-destructive observations (remote sensing). The information collected by the monitoring system can enforce the capability of modeling tools for stability evaluation and hazard assessments.

Therefore a significant element of the strategies for reducing the risk linked to rock landslides is the systematic monitoring of sites which are subject to potential instabilities in order to identify and control the pre-failure movements.

The rock masses are formed by blocks delimited by discontinuities which are characterized using the parameters set by the international society of rock mechanics (ISRM, 1978): attitude, spacing, persistence, roughness, aperture, filling, infiltration. These information have been always collected in the field by skilled operators equipped with instrument able to measure the geometrical and surface characteristic of the rock mass. Because the sections of excavation or natural slopes are often characterized by difficult accessibility, these type of measurements limited and not easily extendable to the whole area of interest. However, thanks to the geometrical nature of these parameters, it is possible to use innovative geomatic techniques, such as laser scanning, for collecting 3D data useful for the automatic or semi-automatic extraction of most of the discontinuity parameters, as described in this work.

This paper describes an approach, based on the terrestrial laser scanner (Laser Scanning Survey - TLS) which represents a valuable alternative to other surveying technique to create rapidly a highly accurate $3 \mathrm{D}$ point clouds to model outcropping rock mass (Pirotti et al., 2013).
Specific processing tools developed for handling and analysing TLS data allows to perform the characterization of rock mass without requiring direct contact with the surface and also providing information of the whole surveyed area.

\section{ROCK DISCONTINUITIES DATA}

When dealing with complex rock masses, the properties of discontinuities are of primary importance, since they determine to large extent the mechanical behavior of the rock mass. The orientation of a discontinuity in space is described by the dip of the line of steepest declination measured from horizontal, and by the dip direction measured clockwise from true north. The orientation of discontinuities largely controls the possibility of unstable conditions or excessive deformations to develop. The importance of orientation increases when other conditions for deformation are present, such as low shear strength and number and distribution of discontinuities. The mutual orientation of discontinuities will determine the shape of the individual unstable blocks.

Traditionally, the measure of the orientation is done with compass and clinometer. Compasses have the advantage that the maximum declination (dip) is measured directly while clinometers need to be moved across the discontinuity wall until the maximum value is registered. When the rock is strongly magnetic a clino-rule and $50 \mathrm{~m}$ tape, or a direct reading azimuth protractor can be used.
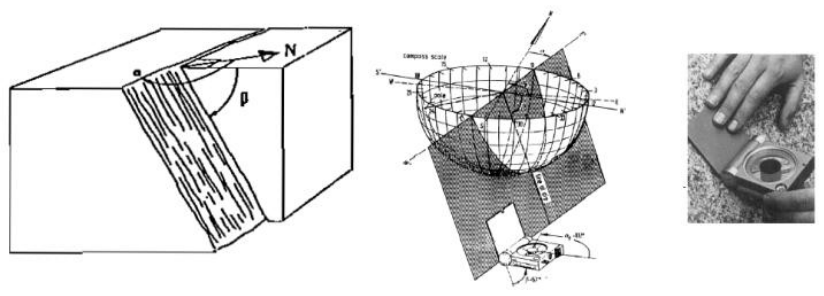

Figure 1. Diagrams indicating the strike, dip and dip direction 
Discontinuity spacing is a basic measurement of the distance between one discontinuity and another. The spacing of the joints (S) is defined as the distance among two planes of discontinuity of a same family, measured in the perpendicular direction to said planes.

Discontinuity spacing determines the dimensions of the blocks of rocks in a slope which influences the overall stability of the slope. Therefore, it is an important parameter in designing appropriate stabilization measures for rock slopes such as rock bolts and rock fall barriers. Similarly, discontinuity spacing is one of the most important parameters to describe the quality of a complete rock mass. It is widely used in the rock mass classification system such as the rock mass rating system.

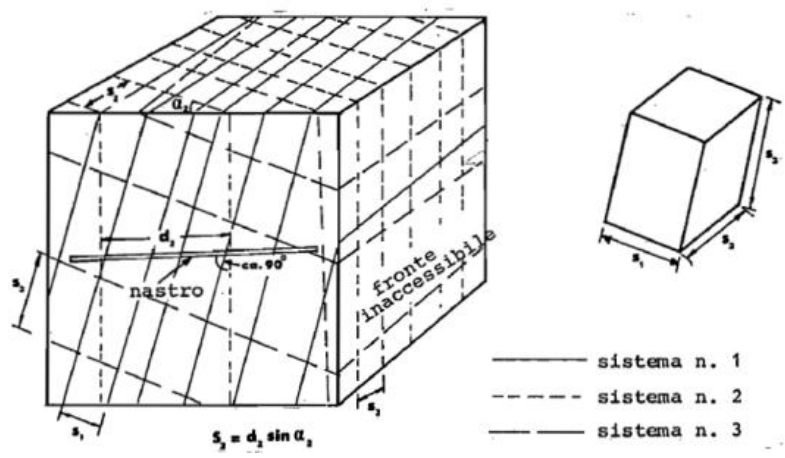

Figure 2. Measurement of joint spacing from observation of a rock exposure

Persistence is the areal extent or size of a discontinuity within a plane. It can be crudely quantified by observing the discontinuity trace lengths on the surface of exposures. It is one of the most important rock mass parameters, but one of the most difficult to quantify by in-situ surveying.

In the case of rock slopes and dam foundations it is of the greatest importance to attempt to assess the degree of persistence of those discontinuities that are unfavorably orientated for stability. The degree to which discontinuities persist beneath adjacent rock blocks without terminating in solid rock or terminating against other discontinuities determines the degree to which failure of intact rock would be involved in eventual failure. The persistence can be, in a simplified way, quantified by observing the length of the visible trace on the free surface.

The smaller the value of the persistence, the less the behavior of the rock mass will be affected by the discontinuity.

\section{SURVEY OF GEOTECHNICAL PARAMETERS WITH DIGITAL TECHNIQUES}

The traditional methods for characterizing a rock mass are well known and validated both from an operational point of view and for the associated accuracy. In the following, we discuss an alternative method based on the use of TLS instruments and dedicated data processing tools for extracting the 3D models and geometrical features useful for the geomechanical surveying.

The data acquisition is based on a laser ranging instrument for the generation of the point clouds coupled to a digital camera for collecting images. Additional surveying instruments (total stations and GNSS receivers) are necessary for georeferencing the point clouds by measuring reflecting targets to be used as Ground Control Points (GCP. Dedicated software tools are adopted for filtering and processing the data by means of manual or semi-automatic approach.

\subsection{D Terrestrial Laser Scan survey technique}

The improvement, over the years, of the 3D surveying sensors and the progress of data processing algorithms have had a strong repercussion on the reconstruction of surfaces. Terrestrial laser scanning is a very effective and realiable surveying technique for collecting dense cloud of 3D points. Different laser scanning systems exist, but the technique used outdoors for geodetic surveying or for measuring large civil engineering structures is mainly based on the "time-of-flight" or "laser range finding" technique (Pirotti et al., 2012). These scanners have a laser diode that sends a pulsed laser beam to the target object. The pulsed laser beam moves through rapidly changing elevation and azimuth angle thanks to a mirror device inside the instrument. The pulse is diffusely reflected by the surface of the scene or object and part of the light returns to the receiver. The time that light needs to travel back and forth from the laser diode to the target is very precisely measured. Knowing the speed of light, the distance from the scanner to the object and the azimuth and angle of the beam, the position of each point where the beam was reflected can be calculated.

The survey yield a digital dataset which is essentially a dense "point cloud" where each point is represented by a set of $3 \mathrm{D}$ spatial coordinate (relative to the internal reference system of the instrument) and the reflected intensity (I) of the laser beam. The most important advantage of the method is that a very high point density can be rapidly collected having a spatial resolution depending on the distance and the rate of acquisition (generally less tha $0.1 \mathrm{~m}$ ) and an accuracy in the order of $0,01 \mathrm{~m}$.

In the context of rock mechanic applications, the most adequate instruments should have a range of acquisition varying between 100 and $1000 \mathrm{~m}$ and a rate of acquisition ranging from 2000 and $100000 \mathrm{pts} / \mathrm{s}$. The accuracy of the $3 \mathrm{~d}$ coordinate decreases with the distance from the object while the density and distribution of the measured points can be influenced by target reflectivity. Optimal results (resolution and density of the digital surface model ) can be obtained by taking several scans of the same object from different points of view.

\subsection{Data processing}

Laser instrument is connected to a portable PC where a software able to set the acquisition, to archive and pre-process the collected data is installed. Based on the setting parameters, mainly related to the area of scanning and to the requested spatial resolution, the instrument acquires the $3 \mathrm{D}$ point cloud in a time span and at a density according to the technical characteristics of the adopted instrument. The acquisition software creates a project for each dataset, which contains the raw data for each scans, the digital images, ancillary information, etc. It also allows a pre-processing step that firstly perform the removal of noise and blunder data filtering. Successively co-registration of different scans, creation of a solid models and integration with other information (mapping of digital images) can be performed. More specifically, the preprocessing step includes the filtering, alignment, georeferencing, segmentation, modeling and texture, that are shown in Figure 3.

The filtering procedure consists in the elimination of the noise and the errors of acquisition. The alignment is a process of recording of all the clouds of points in an unique reference system. This operation results one of the most important and delicate for the correctness of final model. It's possible to perform the alignment through an automatic procedure that 
detect artificial reflecting target by the application of correlation techniques;..

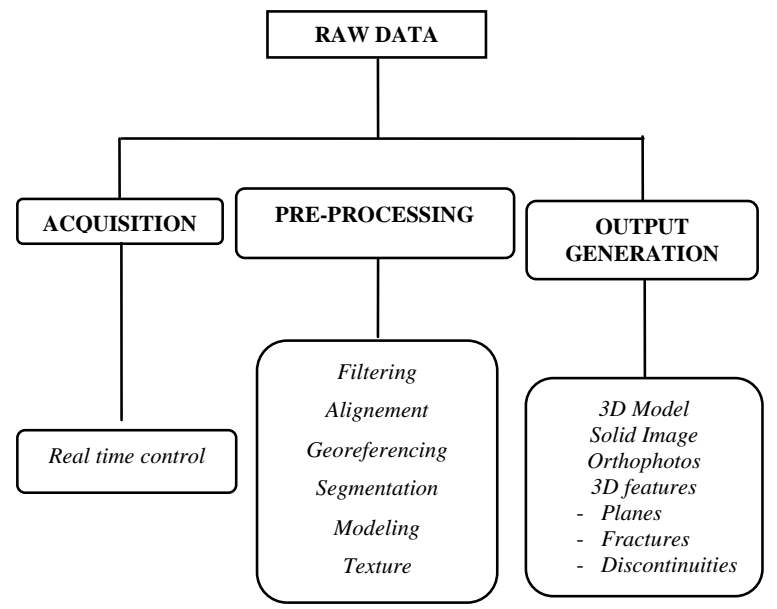

Figure 3. Data management laser

The modeling of surfaces is performed by building a mesh, that consists in the realization of triangles between the points of the cloud and the possibility to generate complex surfaces.

Subsequently, the extraction of parameters of the discontinuities can be performed using an interactive approach through the use of software that allows the manual or automatic selection of the discontinuities to characterize on the acquired images. This operation requires an effort from skilled person to select the most significant areas for which a sufficient number of 3D points are available to estimate the discontinuity features.

In the following examples of test cases conducted for experimenting the procedure described above are presented. A Riegl LMS-Z210ii controlled by RISCAN PRO software was used in order to acquire the point clouds, to perform preprocessing and to extract discontinuity parameters.

\section{CASE STUDIES}

\subsection{The quarries of Torgiovannetto (PG) and Carrara (MS)}

The quarry of Torgiovannetto is located in the limestone of the Maiolica at the northern slopes of Mount Subasio located $2 \mathrm{~km}$ NE of Assisi (PG). The site is interested by instability phenomena both at large (slope movement) and local scale (rock falls of small portion of the front) (Rotonda, T., et al. 2007). The whole front of the quarry was surveyed with the laser scanner and a detailed scan of an area interested by a previous collapse was performed in order to identify the planes of discontinuity (Figure 4) linked to the failure.

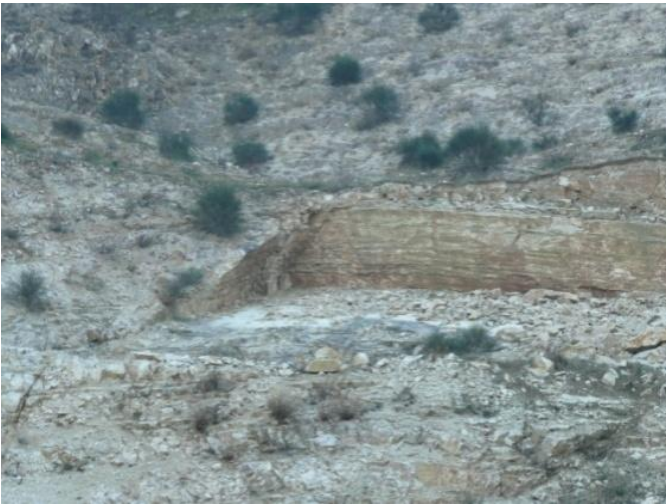

Figure 4. Scar left by the failure at the Torgiovannetto Quarry 2005

After having analysed the area of the collapse, through a accurate comparison between the 3D model and the digital images, we used the software RiSCAN PRO to measure the geometry of each discontinuity: selected points were used to define the orientation of plans bordering the area of the collapse (Figure 5). The coordinates of the baricenter, the direction cosines of the normal to the plane, the direction cosines of the line of maximum slope, dip and dip direction were estimated using RiSCAN PRO tools. In order to verify the capability of the adopted algorithm we tested also on a sample sub-horizontal smooth surface (Figure 6).

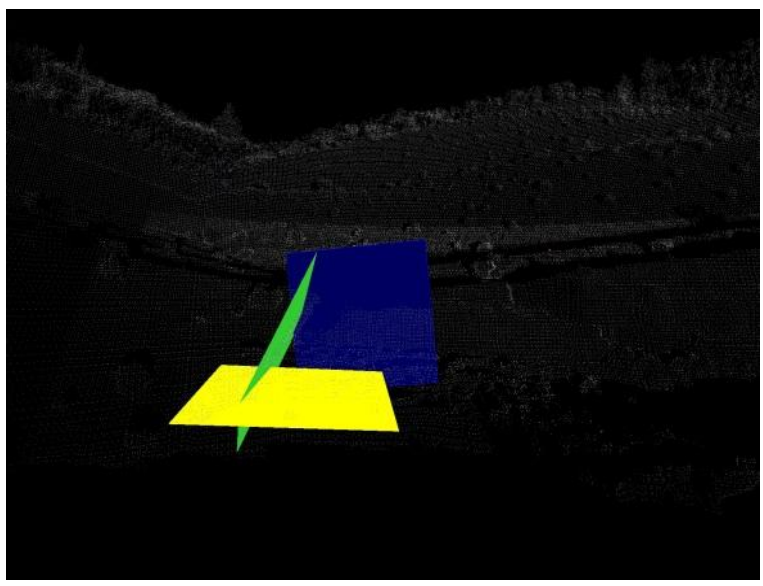

Figure 5. Interpolated plans surrounding the collapsed area

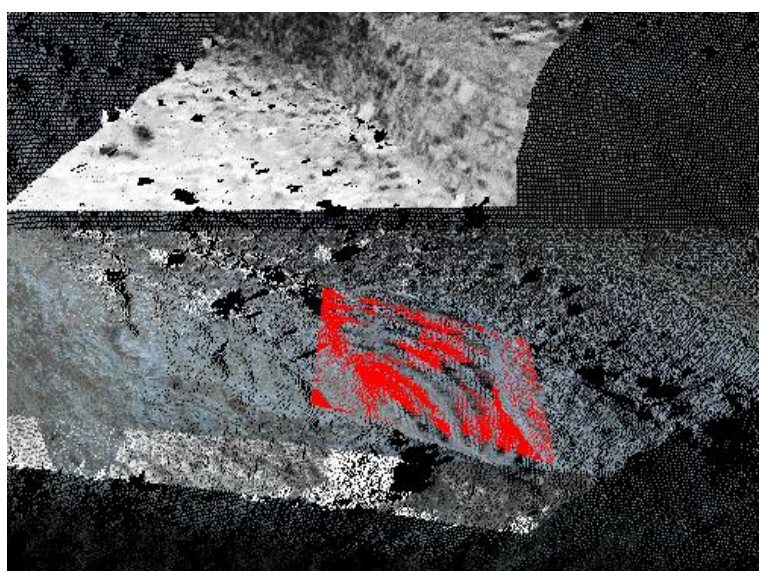

Figure 6. Interpolated smooth surface 
The estimated orientation parameters (Table 1) showed a good agreement (relative error less than $15 \%$ ) with those extracted by a scientific software.

\begin{tabular}{|c|c|c|}
\hline n. & Dip. dir (deg) & Dip. Angle (deg) \\
\hline 6 & 189.6 & 79.4 \\
\hline 7 & 78.4 & 64.5 \\
\hline 8 & 349.0 & 25.6 \\
\hline $\begin{array}{c}\text { Smooth } \\
\text { surface }\end{array}$ & 357.5 & 28.1 \\
\hline
\end{tabular}

Table 1. Estimated orientation parameters

In order to understand the capability of the TLS technique in performing geomechanical survey, the results obtained on a marble quarry located near Carrara (MS) (Rotonda, T., et al. 2007) are reported. In this case, the parameters obtained from a traditional field survey and the same parameters extracted from a point cloud have been compared relatively to large extent of the front. Therefore, the comparison was made in terms of the capability of detecting discontinuity families.

An extract of the results is shown in Table 2 that contains the estimated values for $\operatorname{dip}(\alpha)$ and $\operatorname{dip} \operatorname{direction}(\beta)$ and the associated standard deviations for some of the measured discontinuities. A threshold of $10^{\circ}$ for the dip/dip direction standard deviation was considered in order to remove plans with major approximations.

\begin{tabular}{|c|c|c|c|c|}
\hline Id & $\begin{array}{c}\alpha \\
{\left[{ }^{\circ}\right]}\end{array}$ & $\begin{array}{c}\beta \\
{\left[{ }^{\circ}\right]}\end{array}$ & $\begin{array}{c}{[\sigma(\alpha)]} \\
{\left[{ }^{\circ}\right]}\end{array}$ & $\begin{array}{c}{[\sigma(\beta)]} \\
{\left[{ }^{\circ}\right]}\end{array}$ \\
\hline 3 & 77.8 & 54.4 & 2.3 & 3.4 \\
\hline 5 & 63.3 & 71.6 & 4.7 & 1.4 \\
\hline 7 & 72.3 & 72.4 & 8.6 & 4.5 \\
\hline \hline 9 & 56.2 & 70.2 & 13.8 & 4.1 \\
\hline 12 & 70.6 & 74.1 & 8.0 & 5.4 \\
\hline 16 & 51.5 & 118.4 & 6.1 & 3.8 \\
\hline $\mathbf{H}^{\circ}$ & 56.3 & 70.1 & 13.5 & 3.9 \\
\hline $2 \theta$ & 61.4 & 71.1 & 11.0 & 3.0 \\
\hline 22 & 87.2 & 230.3 & 1.5 & 2.4 \\
\hline
\end{tabular}

Table 2. Example of dip and dip-direction values and their associated standard deviations

Figure 7 shows on an equal-area stereographic projection all the 75 estimated poles of planes.. The spatial distribution of the poles (squares) obtained using the TLS data evidences four sets of discontinuities. TLS mean poles are represented by triangles while circles corresponds to the results from the compass surveys. Comparison between the two methods provided an average angular difference of $10^{\circ}$ for both dip/dip direction values; that taking into account the natural dispersion of the discontinuity families, results can be considered satisfactory.

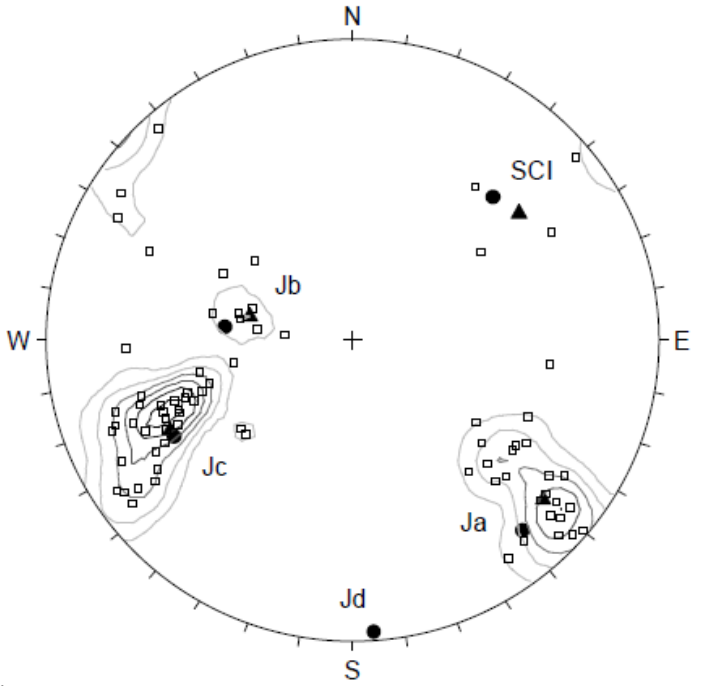

Figure 7. Stereographic projection representing the comparison between the TLS (triangles) and the compass poles (circles) (from Rotonda, T. et al. 2007)

\section{FUTURE DEVELOPMENTS}

The safety of hydroelectric reservoirs founded on rock shoulders is also linked to stability conditions of the surrounding rock slopes. Failures can affect both their storage capacity and can put at serious risks the related infrastructures.

During the last decade, TLS has introduced a new prospective in dam surveying for its ability to detect and monitor the deformation of abutments and to precisely model the structure of the dam. The availability of a 3D detailed model provide useful information especially on aged dam, for which design project are lacking or not enough detailed. Therefore TLS models can be used to implement more refined mathematical methods to understand and simulate the structural behavior and their interaction with the foundation/abutement system.

In 2009 a TLS survey was performed at the Muro Lucano dam (Potenza, Italy) built at the beginning of 1900.

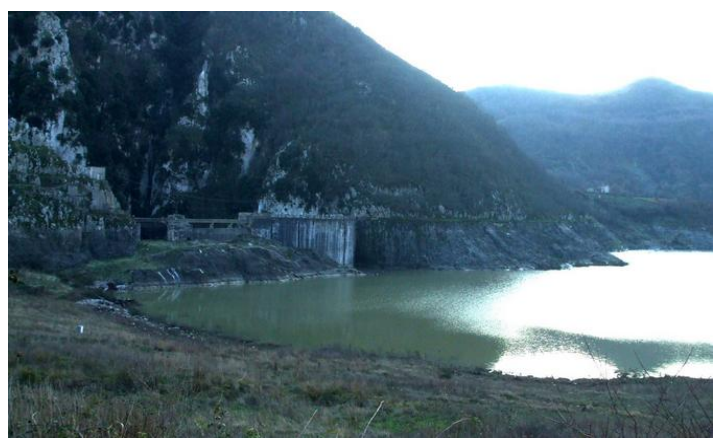

Figure 8. Upstream view of the Muro Lucano (PZ) dam

The structure is an arch-dam $53 \mathrm{~m}$ high (Figure 8) that was never operational because of water infiltrations process considered critical for the dam stability.

A 3D digital model of the structure and the surrounding rock slope was extracted (Figure 9) from data collected with a RIEGL LMS-Z210ii laser scanner,and processed using the RiscanPro software. The survey was performed from three different scan-positionobtaining points cloud that were coregistered using artificial targets and geo-referenced by means of a set of GCPs. 


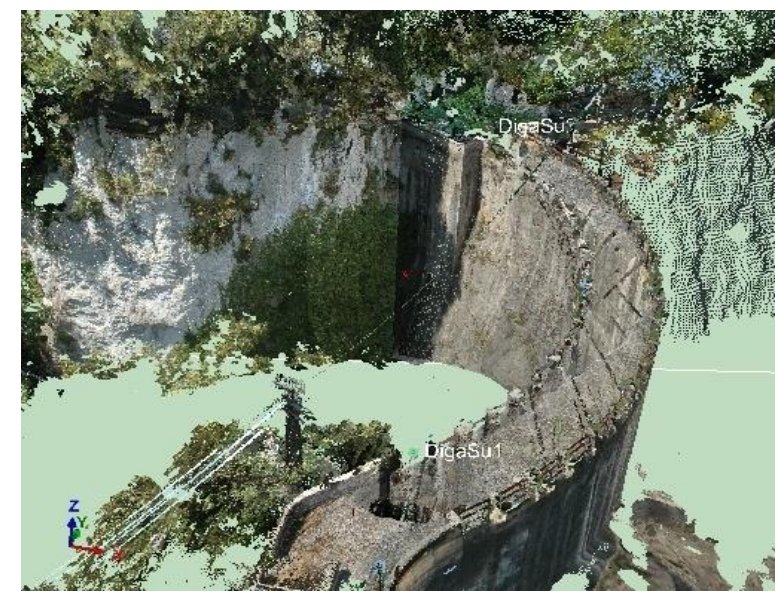

Figure 9. A view of true color 3D point cloud (RGB)

The raw data were filtered to remove the outliers and the points not belonging to the surface of the dam (vegetation, suspended cables, pillars, etc.). Additional information were extracted from the intensity data (backscattering of the reflecting signals) useful to detect deteriorated portions of the surfaces and water infiltrations. The different backscatter that the material, dampened or altered, presents respect to dry/unaltered conditions is registered by the laser sensor as a variation of reflectivity (Table 3). Figure 10 shows an area with different intensity value that corresponds to a water infiltration in the downstream of the dam (left side).

\begin{tabular}{|c|c|c|c|c|c|}
\hline & & \multicolumn{4}{|c|}{ Intensity Value } \\
\hline Material & n. of Point & Min & Max & Mean & St.Dev. \\
\hline $\begin{array}{c}\text { Altered } \\
\text { Concrete }\end{array}$ & 8445 & 0.090 & 0.180 & 0.140 & 0.010 \\
\hline $\begin{array}{c}\text { Unaltered } \\
\text { Concrete }\end{array}$ & 8445 & 0.370 & 0.430 & 0.40 & 0.008 \\
\hline
\end{tabular}

Table 3. statistics of intensity values associated to altered and unaltered surface

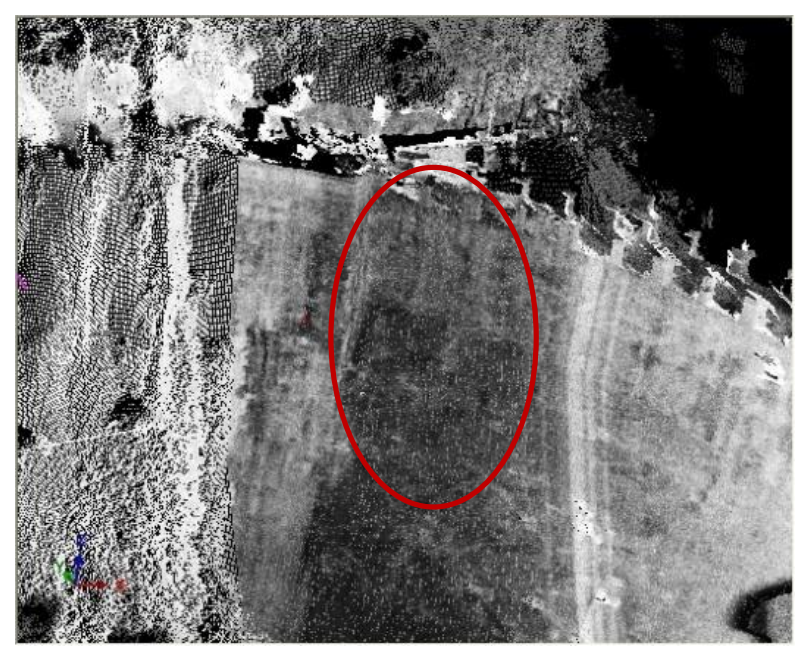

Figure 10. A view of intensity 3D point cloud of the dam. The red circle shows the dam area affected by water infiltration
Finally, TLS provides very detailed point clouds that can be used to characterize unstable rock slopes from geo-mechanical point of view (Figure 11) by measuring the location, the geometry and the volume of the most unstable blocks.

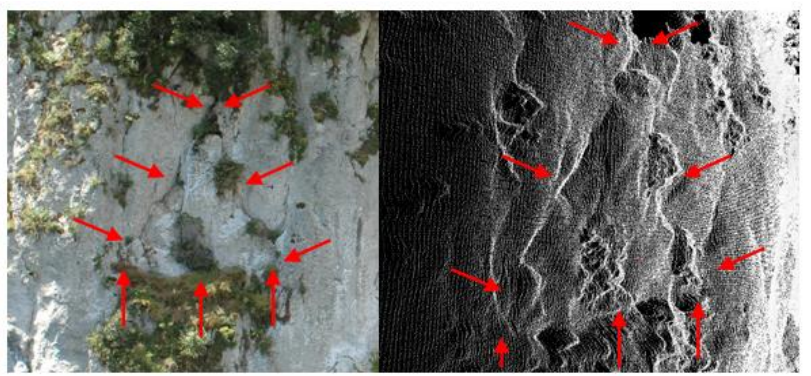

Figure 11. Unstable block identified on a digital image along a rock shoulder of the dam (left) and view of the block on the 3D model rendered using the intensity values

\section{FINAL CONSIDERATIONS}

The presented results illustrate how the use of TLS technique can contribute significantly to the analysis of rock discontinuities. Starting form a point cloud acquired with a laser scanner, it is possible to reconstruct the geometry of the fronts and extract information on both the discontinuity and the state of degradation of the material.

The determination of the planes of discontinuity and their orientation can be obtained using a manual or an automatic procedure. The first approach requires the contribution of expert operators that must define each relevant plane and select a sufficient number of points to determine the dip and dip direction. The second approach is based on automatic or semiautomatic procedures segmentation of the cloud of points in planes. In this work we present results obtained using a the second approach on a limited number of discontinuities compared to reference values obtained from direct surveying. A further improvement is expected from software that extensively perform the tasks of identifying and determining the equations of the planes (Ferrero, A., et al. 2009). Finally, the work illustrate how this technology could be used as a tool for accurately surveying and monitoring large concrete dams and of their rock shoulders. Compared to other innovative techniques that can be employed for dam surveying and monitoring (Corsetti et al. 2013), the use of TLS surveying represents the only method able to acquire 3D data at large distance (up to 300 m) and large areas without significant loss of accuracy.

\section{REFERENCES}

ANIDEL, Le dighe di ritenuta degli impianti idroelettrici italiani - La diga di Muro Lucano vol. V, 137.

Bornaz, L., 2005. L'Analisi ed il trattamento dei dati Laser Scanner terrestri. Ph.D. Thesis in Geodesy and Geomatic. Politecnico di Milano.

Corsetti, M., Nico, G., Di Pasquale, A., Donnarumma, D., Dotti, D., Fiorentino, L., Nicoletti, M., 2013. On the monitoring of dams by means of ground-based radar interferometry (GBSAR), 9th ICOLD European club Symposium on Sharing Experience for Safe and Sustainable Water Storage, Venezia. 
Ferrero, A., Forlani, M., Roncella, G., Voyat, R., 2009. Advanced Geostructural Survey Methods Applied to Rock Mass Characterization. Rock Mech Rock Eng., 42, pp. 631-665.

ISRM, 1978. Suggested methods for the quantitative description of discontinuities in rock masses. Int. J. Rock Mechanics Min. Sci., 15, 319-368.

Kenner, R., Phillips, M., Danioth, C., Denier, C., Thee, P., Zgraggen, A., 2011. Investigation of rock and ice loss in a recently deglaciated mountain rock wall using terrestrial laser scanning: Gemsstock, Swiss Alps, Cold Regions Science and Technology, 67, pp. 157-164.

Pirotti, F., Grigolato, S., Lingua, E., Sitzia, T., Tarolli, P., 2012. Laser scanner applications in forest and environmental sciences. Italian Journal of Remote Sensing, 44(1), pp. 109-123.

Pirotti, F., Guarnieri, A., Vettore, A., 2013. State of the art of ground and aerial laser scanning technologies for highresolution topography of the earth surface. European Journal of Remote Sensing, 46, pp. 66-78.

Priest SD., Discontinuity analysis for rock engineering. London: Chapman \& Hall; 1993.

Rotonda, T., Marsella, M., Lizzadro, L., Ricca, A., 2007. Analysis of laser scanner data collected during a survey of faces in a rock quarry. 11th Int. Congr. on Rock Mechanics, Lisbon.

Schneider, D., 2006. Terrestrial Laser Scanning for area based deformation analysis of towers and water dams. In: Proceedings 3rd IAG / 12th FIG Symposium, Baden. 